\title{
Evolutionary couplings detect side-chain interactions
}

\author{
Adam J Hockenberry ${ }^{\text {Corresp., } 1}$, Claus O Wilke ${ }^{1}$ \\ ${ }^{1}$ Department of Integrative Biology, The University of Texas at Austin, Austin, TX, United States of America \\ Corresponding Author: Adam J Hockenberry \\ Email address: adam.hockenberry@utexas.edu
}

Patterns of amino acid covariation in large protein sequence alignments can inform the prediction of de novo protein structures, binding interfaces, and mutational effects. While algorithms that detect these so-called evolutionary couplings between residues have proven useful for practical applications, less is known about how and why these methods perform so well, and what insights into biological processes can be gained from their application. Evolutionary coupling algorithms are commonly benchmarked by comparison to true structural contacts derived from solved protein structures. However, the methods used to determine true structural contacts are not standardized and different definitions of structural contacts may have important consequences for interpreting the results from evolutionary coupling analyses and understanding their overall utility. Here, we show that evolutionary coupling analyses are significantly more likely to identify structural contacts between side-chain atoms than between backbone atoms. We use both simulations and empirical analyses to highlight that purely backbone-based definitions of true residue-residue contacts (i.e., based on the distance between C $\alpha$ atoms) may underestimate the accuracy of evolutionary coupling algorithms by as much as $40 \%$ and that a commonly used reference point (C $\beta$ atoms) underestimates the accuracy by $10-15 \%$. These findings show that co-evolutionary outcomes differ according to which atoms participate in residue-residue interactions and suggest that accounting for different interaction types may lead to further improvements to contact-prediction methods. 


\title{
Evolutionary couplings detect side-chain interactions
}

\author{
Adam J. Hockenberry ${ }^{1,{ }^{*}}$ and Claus O. Wilke ${ }^{1}$ \\ ${ }^{1}$ Department of Integrative Biology, The University of Texas at Austin, Austin, TX, \\ United States of America
}

7

\author{
Corresponding author: \\ Adam J. Hockenberry* \\ Email address: adam.hockenberry@utexas.edu
}

\begin{abstract}
Patterns of amino acid covariation in large protein sequence alignments can inform the prediction of de novo protein structures, binding interfaces, and mutational effects. While algorithms that detect these so-called evolutionary couplings between residues have proven useful for practical applications, less is known about how and why these methods perform so well, and what insights into biological processes can be gained from their application. Evolutionary coupling algorithms are commonly benchmarked by comparison to true structural contacts derived from solved protein structures. However, the methods used to determine true structural contacts are not standardized and different definitions of structural contacts may have important consequences for interpreting the results from evolutionary coupling analyses and understanding their overall utility. Here, we show that evolutionary coupling analyses are significantly more likely to identify structural contacts between side-chain atoms than between backbone atoms. We use both simulations and empirical analyses to highlight that purely backbone-based definitions of true residue-residue contacts (i.e., based on the distance between $\mathrm{C} \alpha$ atoms) may underestimate the accuracy of evolutionary coupling algorithms by as much as $40 \%$ and that a commonly used reference point ( $\mathrm{C} \beta$ atoms) underestimates the accuracy by $10-15 \%$. These findings show that co-evolutionary outcomes differ according to which atoms participate in residue-residue interactions and suggest that accounting for different interaction types may lead to further improvements to contact-prediction methods.
\end{abstract}

\section{INTRODUCTION}

A long-standing problem in physical biology is to predict the structure of a protein based solely on its amino acid sequence (Anfinsen, 1973; Sadowski and Jones, 2009; Marks et al., 2012). Despite advances in x-ray crystallography (Miao et al., 2015; Batyuk et al., 2016), NMR spectroscopy (Liu et al., 2005; Denisov and Sligar, 2016), cryo-electron microscopy (Liao et al., 2013; Amunts et al., 2014; Punjani et al., 2017), and template-based homology modeling (Söding, 2005; Biasini et al., 2014), the pace at which new structural data is generated pales in comparison to the rate at which we are accumulating new genomes and gene sequences (Rose et al., 2017). As a result, many protein families still lack even a single representative structure or set of functional annotations (Wyman et al., 2018).

In recent years, however, several groups have made substantial improvements to de novo structure prediction by leveraging co-evolutionary information contained within large sequence alignments of protein homologs (Lapedes et al., 1999; Burger and Van Nimwegen, 2008, 2010; Marks et al., 2011; Morcos et al., 2011; Sulkowska et al., 2012; Kamisetty et al., 2013). One key result has been that residues that co-evolve with one another frequently do so as a result of their spatial proximity within the protein structure, i.e. a mutation to one site can be compensated for by subsequent mutations to other directly interacting sites (Gobel et al., 1994; Shindyalov et al., 1994; Weigt et al., 2009). By determining an "evolutionary coupling" score for all pairs of amino acid residues within a sequence alignment-and assuming that the highest-scoring residue-residue pairs are in close spatial proximity within the structurethe search space of computational protein folding methods can be constrained, resulting in accurate 3D-structure determination (Marks et al., 2011; Hopf et al., 2012; Ovchinnikov et al., 2017). Other applications have used evolutionary coupling scores to predict protein binding partners and interfaces 
(Burger and Van Nimwegen, 2008; Hopf et al., 2014; Ovchinnikov et al., 2014), as well as to predict the effect of mutations on protein stability and function (Hopf et al., 2017). Many of these approaches have been further improved through the use of machine learning (Cheng and Baldi, 2007; Jones et al., 2015; Michel et al., 2017), and specifically deep neural networks that leverage evolutionary couplings along-side numerous other protein features (Tegge et al., 2009; Lena et al., 2012; Xiong et al., 2017; Stahl et al., 2017; He et al., 2017; Wang et al., 2017; Riesselman et al., 2018; Liu et al., 2018; Wozniak et al., 2018; Jones and Kandathil, 2018; Adhikari et al., 2018; Hanson et al., 2018).

During the development and testing of evolutionary coupling algorithms, predictions of residueresidue couplings from large multiple-sequence alignments are frequently benchmarked against a set of known protein structures to determine their accuracy in identifying residue-residue contacts (Figliuzzi et al., 2018; Schaarschmidt et al., 2018; Wang et al., 2018). The large number of protein structures that have been solved at atomic resolution provides a training dataset where intramolecular contacts are known (Rose et al., 2017). However, even the most high-resolution crystal structures of proteins require extrapolation from the location of particular atoms and residues to classify residue-residue contacts (Seeliger and de Groot, 2007; Sathyapriya et al., 2009; Duarte et al., 2010; Yuan et al., 2012). A commonly used heuristic is to determine that any amino acid residue that lies within some pre-defined physical distance - frequently $8 \AA$ - of another amino acid residue is said to be in structural contact (Marks et al., 2011).

Different reference points can be used when determining the distance between any pair of amino acid residues, and prior research has shown that the choice of reference point can matter in some contexts. For example, in the context of structural determinants of sequence conservation, it has been found that sequence conservation is more strongly correlated to the number of residue-residue contacts identified via side-chain centers than identified via $\mathrm{C} \alpha$ atoms (Lin et al., 2008; Marcos and Echave, 2015; Shahmoradi and Wilke, 2016). However, it is unknown whether the choice of reference point is important for evaluating and interpreting modern evolutionary coupling approaches.

Additionally, some studies eschew fixed reference points entirely. Residue-residue contacts can be defined according to whether the minimum distance between all possible pairs of heavy atoms between two residues falls below a set distance cutoff (Ovchinnikov et al., 2017). However, it is unclear whether all atoms should be considered in this calculation or whether backbone and side-chain atoms should be treated differently. Further, there are a number of ever more complex approaches that can be used to define contacts given a protein structure, including the use of hydrogen bonding and residue interaction networks (Doncheva et al., 2011; Bhattacharya and Cheng, 2013; Piovesan et al., 2016), as well as correlated residue movements from molecular dynamics simulations (Scarabelli and Grant, 2013; Doshi et al., 2016; Serçinoğlu and Ozbek, 2018).

Currently, there are no accepted standards in the field for how to determine a set of residue-residue contacts for a given protein structure. Further, there has yet to be a systematic analysis of whether co-evolutionary signatures are more- or less-closely related to different types of intramolecular contacts that may exist within a given structure. Here, we systematically test the accuracy of several evolutionary coupling algorithms against true positive contacts defined via $\mathrm{C} \alpha, \mathrm{C} \beta$, or side-chain geometric centers, as well as via minimum distances between all atoms or atoms residing in side chains only. We find that residue-residue contacts defined according to side-chain centers and side-chain atoms are much more accurately predicted by evolutionary couplings. These results imply that the dominant epistatic effects resulting in co-evolutionary signatures arise from interactions between side-chain atoms. Our findings highlight the importance of the choice of contact definition and provide insight into the constraints governing the evolution of protein structures.

\section{MATERIALS AND METHODS}

\section{Dataset compilation and processing}

We downloaded the so-called PSICOV dataset of 150 proteins that have been extensively studied (Jones et al., 2012, 2015; Jones and Kandathil, 2018). We processed each starting PDB file to select a single chain, ensure a consistent numbering of residues $(1 \ldots n)$, test for unknown or non-standard residues, select the most likely state for all disordered sequence atoms, and remove all extraneous information (including "HETATM" lines). Next, to ensure that all residues were represented in full and repair those that were not, we used PYROSETTA to read in the ".PDB" files using the 'pose_from_pdb' function and wrote the output as our final clean structure (Chaudhury et al., 2010). 


\section{Determining structural contacts and contact-types}

From each cleaned ".PDB” file, we calculated residue-residue distance matrices using custom python scripts (the euclidean distance from 3-dimensional atomic coordinates). All residues contain a $\mathrm{C} \alpha$ atom so this calculation was straightforward. For $\mathrm{C} \beta$ calculations, we used the $\mathrm{C} \beta$ atom of all residues except glycine, for which we continued to use the $\mathrm{C} \alpha$ atom. For side-chain center calculations, we calculated the geometric center of each residue based on the coordinates of all non-backbone heavy atoms. This calculation included $\mathrm{C} \beta$ atoms but excluded $\mathrm{C} \alpha$ atoms for all amino acids except glycine, for which we again continued to use $\mathrm{C} \alpha$ as the reference point.

To calculate minimum atomic distances between two residues, we calculated all pairwise euclidean distances between heavy atoms and selected the minimum distance. In extending this analysis to only consider side-chain atoms, we continued to consider $\mathrm{C} \beta$ atoms as part of the side chain but not $\mathrm{C} \alpha$. Again, we relaxed this restriction for glycine and included $\mathrm{C} \alpha$ as a side-chain atom to permit calculations for this amino acid.

For all methods, contacts were assessed by first removing all residue-residue pairs where the two residues were shorter than 12 amino acids apart in chain distance. Contacts were determined throughout this manuscript for each structure according to an $8 \AA$ cutoff between $\mathrm{C} \alpha$ atoms. Since accuracy values are partially dependent on the number of true positives that are called, we maintained a constant number of true positive contact classifications throughout to facilitate comparison between methods. For each contact definition ( $\mathrm{C} \beta$, side-chain center, minimum atomic distances), we selected $n$ residue-residue pairs with the shortest distances where $n$ is the number of contacts defined according to the aforementioned $\mathrm{C} \alpha$-based method. Thus, the actual distance cutoff used to classify contacts for these other metrics varies slightly from protein to protein.

To classify residue-residue pairs ( $a$ and $b$ ) via their side-chain orientations, we chose a reference residue ( $a$ ) and drew two vectors: i) from the $\mathrm{C} \alpha$ atom coordinate to the side-chain center for that residue and ii) from the $\mathrm{C} \alpha$ atom coordinate to the $\mathrm{C} \alpha$ atom coordinate for the other residue in question $(b)$. If the angle between these two vectors was less than $\pi / 2$ radians, the side chain of residue $a$ was said to point towards residue $b$. To determine the residue's classification we next repeated the calculation using residue $b$ as the reference and finally classified the residue-residue pair accordingly.

\section{Evolutionary coupling algorithms}

For each of the 150 proteins in our dataset, we followed a principled method to retrieve homologous sequences. We first extracted the amino acid sequence from the ".PDB" file. We next used PHMMER to search through progressively larger databases in order to retrieve up to 10,000 homologous sequences (Potter et al., 2018). To do so, we downloaded local versions of the rp15, rp35, rp55, and rp75 databases (Chen et al., 2011). We first searched the smallest, least redundant, database for each sequence using an E-value threshold of 0.0001. For any sequence with greater than 10,000 hits we stopped and selected the top scoring 10,000 hits for further analysis. For sequences with fewer than 10,000 hits we moved to the next largest database and repeated the process. Finally for the small number of sequences for which we did not accumulate at least 1,000 sequences in the largest database (rp75), we used the online version of PHMMER to search the UniprotKB database and downloaded the maximum results.

For each protein, we next aligned the hits along-side the reference sequence using MAFFT (v7.380, L-INS-i method with default parameters) (Katoh and Standley, 2013). Next, we cleaned these results to remove all columns that were gapped in the reference (“.PDB”) sequence. All other columns and sequences in the sequence alignments were retained regardless of gap coverage.

Using these alignments, we next calculated evolutionary couplings between residue-residue pairs. All results in the main manuscript are displayed using CCMpred with default parameters ( 0.8 local sequence re-weighting threshold, 0.2 pairwise regularization coefficients, average product correction) (Seemayer et al., 2014). We additionally used the 'plmc' method from the EVcouplings framework with default parameters (no average product correction) (Hopf et al., 2018) and PSICOV (default parameters excepting: “-z 50 -r 0.001") (Jones et al., 2012) to ensure the robustness of our findings.

Except where otherwise noted, main text results were calculated using the top $L / 2$ couplings for each protein where $L$ is the length of the reference amino acid sequence. Positive Predictive Value (PPV) is calculated as the number of classified contacts among these top couplings divided by the total number of top couplings considered. 

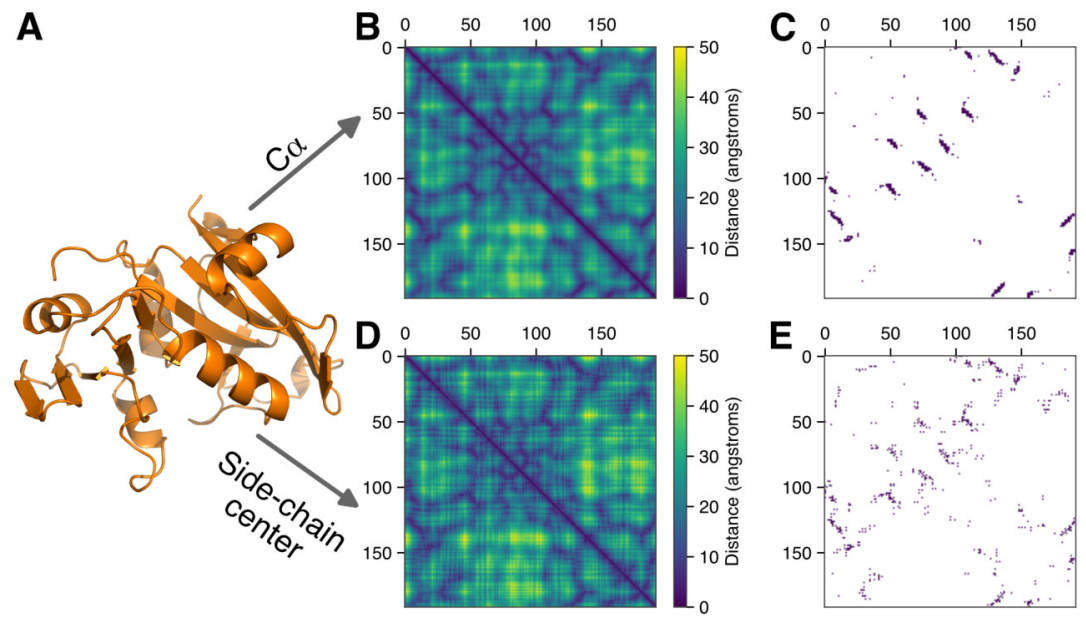

Figure 1. Constructing contact maps from protein structures. (A) An example structure (PDB:1AOE). (B) A symmetric distance matrix between all pairs of amino acid residues measured from each residue's $\mathrm{C} \alpha$ atom. (C) Medium- to long-range contacts $(\geq 12$ residues apart along the linear chain are identified using an $8 \AA$ cutoff (dark blue). (D) and (E) Same methodology as depicted in (B) and (C), using the geometric center of each residue's side chain as a reference point for measuring distances.

\section{Evolutionary simulations}

For the example protein used throughout the text (PDB:1AOE) we performed mutation accumulation simulations using PYROSETTA (Chaudhury et al., 2010). We first read in the ".PDB" structure (with disulfide bonds disabled), and minimized it to optimize thermodynamic stability via rotamer selection and backbone movements. We next fixed the backbone, and implemented an expedited evolutionary algorithm to select amino acid point mutations (no insertions or deletions were allowed) according to their predicted impact on structural stability (Teufel and Wilke, 2017; Jiang et al., 2018). At each step, we selected a random amino acid position, and attempted to mutate it randomly to one of the remaining 19 amino acids. We re-packed the structure within a $12 \AA$ radius and determined whether or not to accept the mutation based off of the resulting change in structural stability. Mutations which either did not alter or increased stability (i.e. resulted in a decreased $\Delta G$ ) were accepted with a probability of 1. Mutations that decreased stability were accepted with a probability proportional to their $\Delta \Delta G$ as in Teufel and Wilke (Teufel and Wilke, 2017). At the end of the evolutionary process, the resulting amino acid sequence was stored for future analysis.

We performed thousands of independent replicates of this expedited evolutionary process where we altered the number of accepted mutations that we accumulated before halting the simulation, the number of replicate evolutionary experiments that we performed, and the fraction of the initial wild-type stability value that we used for our selection criteria. Collections of the resulting sequences were analyzed via evolutionary coupling algorithms in the same manner as empirical sequences, with no need for sequence alignment.

\section{RESULTS}

\section{Structural contact definitions}

Putatively true interactions between amino acid residues within a given protein family are frequently derived from the distance between residues in a representative protein structure. Figure 1 depicts an example protein structure (PDB:1AOE) alongside two symmetric matrices depicting all residue-residue distances (in angstroms, $\AA$ ) defined according to either the distance between the $\mathrm{C} \alpha$ atoms of individual residues or the distance between the geometric centers of each residue's side chain. We use the geometric center of a residue's side chain throughout this manuscript and note that, unlike the center-of-mass, this metric includes only information about the spatial coordinates of atoms and is thus agnostic to their identity/mass.

From these residue-residue distance matrices, we created contact matrices by using an $8 \AA$, C $\alpha$-based 
distance cutoff to define contacts for a given protein. To facilitate a direct comparison between methods, we use the same absolute number of contacts to determine a comparable distance cutoff (specific to each protein) to use for side-chain center distances such that an equal number of true contacts were identified regardless of the reference point used to measure distances. For instance, in Fig. 1 we found that the $8 \AA, \mathrm{C} \alpha$-based distance cutoff resulted in 295 contacts. We thus chose the distance cutoff for side-chain centers such that 295 contacts were identified, corresponding to a distance cutoff of $7.33 \AA$ for this example protein. Additionally, for most applications the structurally interesting contacts are those of amino acids that are not close to each other in the linear sequence. We define those here as amino acid pairs separated by a chain distance of at least 12 residues, and we only consider this subset of possible contacts for this example and the remainder of this manuscript.

While the distance matrices look similar for an example protein when calculated via $\mathrm{C} \alpha$ atoms or side-chain centers (Fig. 1B compared to D), the resulting maps of residue-residue contacts show considerable heterogeneity (Fig. 1C compared to E). More quantitatively, the set of all residue-residue distances measured by $\mathrm{C} \alpha$ atoms are highly correlated with comparable distances measured via side-chain centers (Fig. 2A). However, this strong overall correlation obscures important differences in contact definitions which we observe when focusing within the narrow region where direct amino acid residue contacts are defined (Fig. 2B). For 1AOE, we identified a total of 295 contacts according to the $8 \AA$ $\mathrm{C} \alpha$-based distance cutoff. Of the shortest 295 contact distances identified via side-chain centers, the percent of residue-residue pairs that appear in both definitions is only $56 \%$.

To assess the generality of these findings, we applied this analysis to a commonly used benchmark set of 150 proteins (Jones et al., 2012, 2015; Jones and Kandathil, 2018). Across all of these proteins, we observed a median correlation of 0.97 between residue-residue distances calculated via $\mathrm{C} \alpha$ atoms

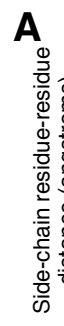

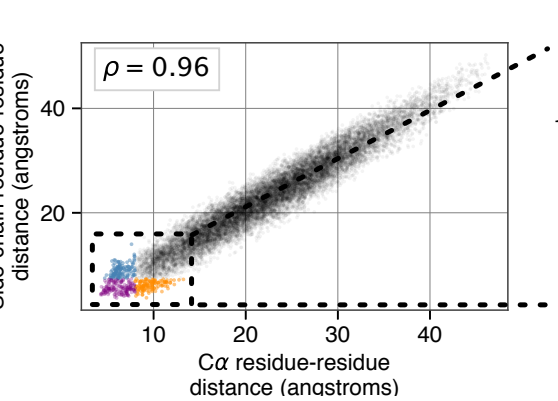

B

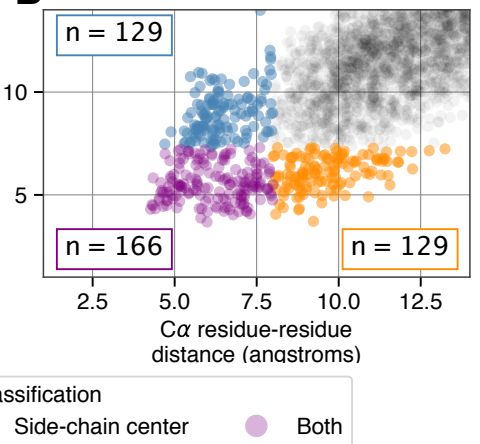

\section{C}
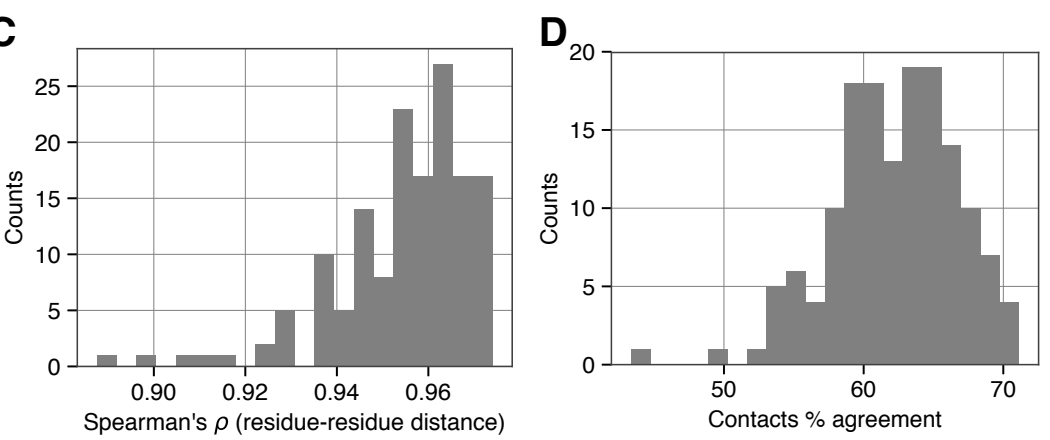

Figure 2. Relationship between different contact identification methods. (A) Correlation between residue-residue distances in PDB:1 AOE measured according to $\mathrm{C} \alpha$ atoms and side-chain centers. $(B) \mathrm{A}$ zoomed in view (right) highlights variably defined residue-residue contacts indicated by the various colors. Cutoffs for defining contacts are $8 \AA$ and $7.33 \AA$ for the $\mathrm{C} \alpha$ and side-chain center based metrics for this protein. $(C)$ Distribution of Spearman's correlation coefficient values $(\rho)$ between residue-residue distances for 150 different proteins. $(D)$ Distribution of the percent agreement for contact definitions for the same set of proteins. (SI Fig. S2 shows a comparable comparison between $\mathrm{C} \beta$ and side-chain center distances.) 
and side-chain centers (Fig. 2C), but a median overlap of just 63\% between contacts defined via $\mathrm{C} \alpha$ and side-chain centers (Fig. 2D). The distribution of protein-specific distance cutoffs that we used to identify equal numbers of contacts across methodologies was approximately normal and in the range of 6-8 Ås (SI Fig. S1).

We focused on comparing the use of $\mathrm{C} \alpha$ atoms and side-chain centers as reference points to determine contacts, since these two features represent extreme ends of the spectrum. In practice, $\mathrm{C} \beta$ atoms occupy an intermediate location between the backbone and the side-chain center and are one of the most frequently used reference points (Seeliger and de Groot, 2007; Sathyapriya et al., 2009; Duarte et al., 2010; Yuan et al., 2012). We repeated the primary results of this section by comparing the use of $\mathrm{C} \beta$ atoms and side-chain centers as reference points and observed only a 78\% overlap in residue-residue contacts identified by these two methods (SI Fig. S2). Together, these findings highlight that true contacts vary substantially according to the reference point used to measure residue-residue distances.

\section{Evolutionary couplings reflect side-chain contacts in simulated sequence alignments}

While the previous analysis of empirical structures shows that the choice of reference point has important consequences for true contact identification, it is not clear which of the different methods is more biologically correct or practically meaningful. We thus turned our attention to a simplified biophysical system of simulated protein evolution to test the ability of evolutionary coupling analyses to recover intramolecular contacts. We used the ROSETTA modeling software (Chaudhury et al., 2010; Leaver-Fay et al., 2011; Kellogg et al., 2011) to perform all-atom simulations of the evolutionary process (Teufel and Wilke, 2017; Jiang et al., 2018) while selecting for the maintenance of protein stability (expressed as a fraction of the initial PDB model stability). We simulated thousands of independent evolutionary trajectories and used the resulting amino acid sequences from these simulations to calculate evolutionary couplings using CCMpred with default parameters (Seemayer et al., 2014). Within this defined system, we are able to remove the constraints of phylogenetic biases, limited data availability, homo-oligomerization, insertions/deletions, and changes in evolutionary pressures over time between species-all of which partially limit the power of algorithms to detect true evolutionary couplings in real data (Anishchenko et al., 2017).

We continued to use $1 \mathrm{AOE}$ as an example structure and varied several parameters of our simulation to ensure robust results. We defined true positive residue-residue contacts according to the original PDB structure using residue-residue distances calculated between different quantities for comparison ( $\mathrm{C} \alpha, \mathrm{C} \beta$, and side-chain center with respective distance cutoffs of $8 \AA, 7.5 \AA$, and $7.33 \AA$ ). To assess the accuracy of evolutionary couplings, we determined the positive predictive value (PPV) of the top $L / 2$ couplings-where $L$ is the chain length of the protein under investigation, $L=192$ in the case of $1 \mathrm{AOE}$. For C $\alpha$-based contact definitions, we found that the PPV increases rapidly according to the number of independent sequences that we simulated and consequently used as input for evolutionary coupling analyses (Fig. 3A). For these simulations, we ran each independent evolutionary simulation until we accepted a number of point mutations totaling 10 times the length of the protein sequence. However, regardless of the selection strength that we imposed on the sequence evolution (colored lines in Fig. 3A), PPV values plateaued at a value below 0.75 . This indicates that more than a quarter of the top ranked evolutionary couplings represent incorrect predictions and are not true intramolecular contacts. By contrast, when we analyzed the same evolutionary coupling values but instead used side-chain center distances to define true contacts, we observed that nearly all of the top ranked couplings were defined as true contacts (PPV values approached 1).

We additionally explored how the number of mutations accumulated per sequence affected the ability of evolutionary coupling algorithms to recover intramolecular contacts. We fixed the number of replicate sequences at 3000, and found that PPV values showed minimal variation according to the number of accepted mutations that had accumulated per sequence over the course of our in silico evolution (Fig. 3C). As before, however, prediction accuracies were substantially higher when we defined true contacts according to side-chain center distances (Fig. 3D). These simulation results highlight thatacross numerous parameter combinations- the top $L / 2$ evolutionary couplings corresponded to true intramolecular contacts as long as true positives were defined according to side-chain centers and not $\mathrm{C} \alpha$ carbons. Definitions of contacts based on $\mathrm{C} \beta$ atoms resulted in intermediate accuracy, plateauing at higher values than $C \alpha$ but lower than side-chain centers (SI Fig. S3). 

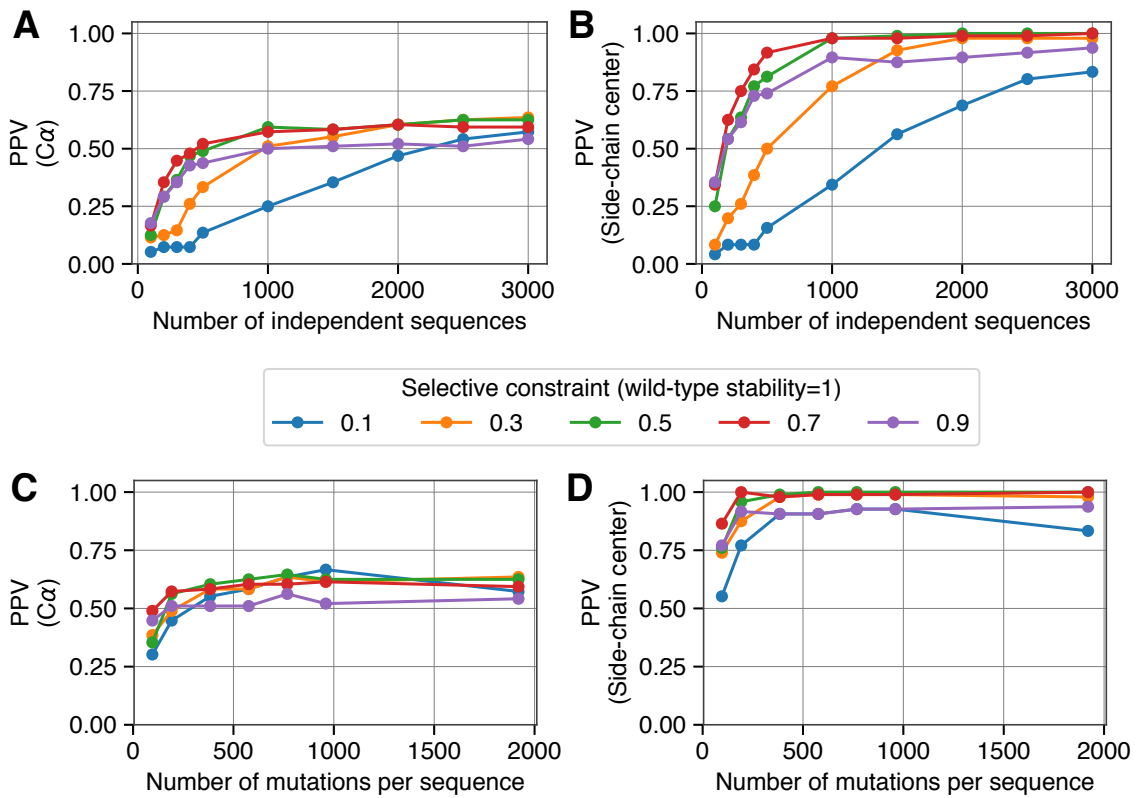

Figure 3. Comparing simulation-derived evolutionary couplings to different contact definitions. (A) For each of 5 separate selection strengths (colored lines), we ran evolutionary simulations until a number of mutations totaling 10 times the length of the protein were accumulated per replicate. We varied the number of independent replicate sequences (x-axis) that were used as input for evolutionary coupling analysis, and found that the resulting evolutionary couplings fail to fully recover $\mathrm{C} \alpha$ defined contacts $(8 \AA ̊)$ for PDB:1AOE. $(B)$ By contrast, contacts defined via side-chain centers $(7.33 \AA$ ) are near-perfectly recovered across a range of simulation parameters. $(C)$ and $(D)$ Similar to parts (A) and (B), but along the $\mathrm{x}$-axis we now show results from simulations where a different number of accepted mutations were accumulated per sequence. We fixed the number of replicate sequences that were simulated — and used as input for evolutionary coupling analysis-at 3,000 for each of these data points. (Results comparing $\mathrm{C} \beta$ and side-chain center contact definitions, can be found in SI Fig. S3.)

\section{Evolutionary couplings reflect side-chain contacts in natural sequence alignments}

To see how evolutionary couplings compare to different definitions of true residue-residue contacts in empirical data, we used PHMMER to identify sequence homologs for each of the 150 proteins (see Materials and Methods for details). We assessed the relationship between evolutionary couplings and structural contacts for all proteins by calculating the positive predictive value (PPV) of the highest $L / 2$ couplings.

As expected, the PPV between the top $L / 2$ evolutionary couplings and $C \alpha$-based contacts varied substantially across the 150 structures. This variation may result from a number of different effects, and we observed a clear correlation between PPV values and the number of homologous sequences used to determine evolutionary couplings (SI Fig. S4). Despite the variability in prediction accuracy between proteins, we observed systematic differences in the PPV according to which metric was used to identify true positive contacts from the PDB structure files (Fig. 4A). When compared to $\mathrm{C} \alpha$ based distances, residue-residue distances measured according to $\mathrm{C} \beta$ atoms resulted in significantly higher PPVs, and side-chain based contact distances resulted in even further improvements (Wilcoxon signed-rank test, $p<10^{-20}$ for all comparisons). Further, the magnitude of the effect was substantial: across all 150 proteins the median percent increase in PPV between $\mathrm{C} \alpha$ and side-chain center contact identification methods was $43 \%$ (Fig. 4B,C). Even between the more similar $\mathrm{C} \beta$ and side-chain center methods, the median percent increase in accuracy was $13 \%$ (Fig. 4D,E). Both comparisons were highly significant and persisted across the entire range of PPVs represented within our dataset (Fig. 4B,D). Additionally, these results were highly consistent across different evolutionary coupling algorithms (SI Figs. S5 \& S6).

We also considered an alternative method for computing contacts: determining structural contacts based on the minimum distance between any two atoms belonging to different two different residues 
(Ovchinnikov et al., 2017). We implemented two versions of this algorithm, determining the minimum distance between: i) all heavy atoms within residues and ii) side-chain heavy atoms only. In each case, and to continue to facilitate a direct comparison between methods, we selected the $n$ shortest distances as contacts where $n$ is the number of contacts identified for each protein via the $8 \AA$ distance cutoff using $\mathrm{C} \alpha$. The resulting PPVs were significantly higher when contacts were defined only according to side-chain atoms as opposed to the complete set of backbone and side-chain atoms (Fig. 4A). We further note that PPVs calculated via side-chain center distances were statistically indistinguishable from PPVs derived from the minimum distance between all heavy atoms within side chains.

All analyses thus far have been performed using a variable distance cutoff to identify true contacts, such that an equal number of true contacts were identified for each structure regardless of the method. This choice was made to facilitate the comparison of PPV values across different contact identification methods. If one contact identification method, for instance, were to identify half the number of true contacts for a given structure compared to another method, the expected PPV would be very different and thus PPV values could not be meaningfully compared without further normalization or re-scaling. However, it would nevertheless be useful to have a uniform distance cutoff to apply to all structures and such a uniform cutoff is more physically realistic and practically useful. We thus repeated our analysis after fixing the $\mathrm{C} \beta$ and side-chain center distance cutoffs at 7.6 and 7.5 $\AA$ s, respectively (values chosen as being close to the median for the distributions displayed in SI Fig. S1).

For $\mathrm{C} \alpha, \mathrm{C} \beta$, and side-chain center methods, there was no significant difference in the number of contacts identified within each structure (SI Fig. S7A) and the conclusions of Fig. 4 with regard to PPV remain the same (SI Fig. S8). However, for comparisons involving the minimum distance between atoms, we found that considering side-chain atoms only resulted in substantially fewer true contacts (using a distance cutoff of $4.5 \AA$ between any two heavy atoms to define contacts, SI Fig. S7A) and the raw PPVs between these two methods cannot be meaningfully compared. By contrast, the average precision score (the weighted mean of PPV across all recall thresholds, similar in spirit to the area under the precision-recall curve) is another measure of accuracy in binary classification tasks and is less sensitive to variability in the number of true positives. We thus compared the average precision scores for all methods using fixed distance cutoffs and found that contacts identified based on side-chain centers resulted in the highest average precision score. Further, considering only the side-chain atoms when looking at the minimum atomic distance resulted in significantly higher scores compared to considering all atoms (SI Fig. S7B). Taken together, this analysis of fixed distance cutoffs is more physically meaningful than our previous approach that used protein-specific cutoffs to facilitate statistical comparisons, and the results support the finding that evolutionary couplings are largely reflective of side-chain based residue-residue interactions.

\section{Side-chain orientations are important to consider when examining residue-residue in- teractions}

The preceding analyses have shown that for the exact same evolutionary couplings, PPVs are substantially higher when using side-chain based distances to identify true positive intramolecular contacts than when using either $\mathrm{C} \alpha$ or $\mathrm{C} \beta$-based distances. To look more specifically at why these differences were so pronounced, we decided to investigate the orientation of residue-residue pairs identified by the various criteria. Any two residues considered to be in contact can display one of three distinct orientations of their respective side chains: i) both residues' side chains may point towards one another with the energetic interactions occurring through side-chain atoms, ii) one residue's side chain may point towards the other residue while that residue's side chain points away, or iii) both residues' side chains may point away from one another with energetic interactions occurring between the respective amino acid backbones (Fig. 5A). As we expected, the $\mathrm{C} \alpha$-based definition yields fractions close to the random expectation for $1 \mathrm{AOE}$ : in $\sim 25 \%$ of the cases side chains point towards each other, in another $\sim 25 \%$ of the cases they point away from each other, and in the remaining $\sim 50 \%$ of the cases one side chain points towards the other while the other points away (Fig. 5B). By contrast, side-chain (and to a lesser degree $\mathrm{C} \beta$ ) based contact definitions enrich for cases where both side chains point towards one another (Fig. 5C,D).

Across all 150 proteins in our dataset, we calculated the fraction of all residue-residue pairs (regardless of distance in 3D space but subject to the same chain distance constraints applied throughout this manuscript) where both side chains point towards one another and found a median of 0.2 (Fig. 5E, "All pairs"). However, this fraction increases progressively when we consider residue-residue pairs identified 

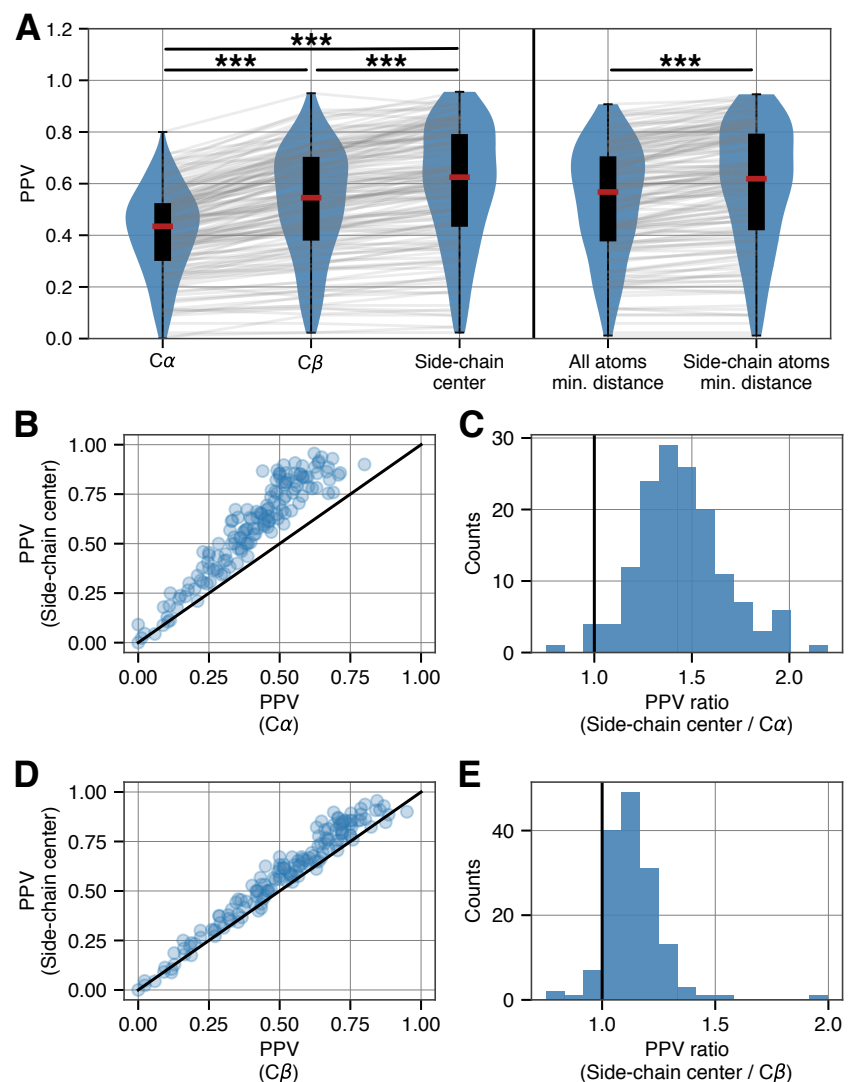

Figure 4. Accuracy of evolutionary couplings derived from empirical alignments. $(A)$ For a diverse set of 150 proteins, the PPV of the top $L / 2$ evolutionary coupling scores-derived from empirical sequence alignments - is progressively higher when intramolecular contacts are defined according to C $\alpha$ atoms, $\mathrm{C} \beta$ atoms, and side-chain centers. Similarly, PPVs are higher when computing contacts based on side-chain atoms only as opposed to considering all possible interactions between atoms in residues. (*** indicates $p<10^{-20}$, Wilcoxon signed-rank test) $(B)$ Scatter plot of PPVs for each protein according to $\mathrm{C} \alpha$ and side-chain center contact identification methods. $(C)$ Histogram of the ratios from the data in (B) indicate that the median percent increase in accuracy is $43 \%$. (D) and (E) As in (B) and (C), but now comparing $\mathrm{C} \beta$ and side-chain center contact identification methods. Results show a median percent increase in contact identification accuracy of $13 \%$. (Results for two other evolutionary coupling algorithm implementations can be found in SI Figs. S5 \& S6. Further analyses performed with fixed distance cutoffs can be found in SI Figs. S7 \& S8.) 
as true contacts for each protein according to $\mathrm{C} \alpha, \mathrm{C} \beta$, and side-chain centers-illustrating that the trend observed in (Fig. 5B-D) applies broadly. If instead we consider residue-residue pairs predicted by the top ranked evolutionary couplings, we observe that a large fraction of these couplings are between residues whose side chains point towards one another in the reference protein structure. Additionally, this fraction is highest for the most highly ranked evolutionary couplings (Top $0.25 \mathrm{~L}$, where $\mathrm{L}$ is the length of the protein) and is substantially higher than the proportion identified by $\mathrm{C} \alpha$-based distances (Fig. 5E).

This analysis of side-chain orientations highlights that strong evolutionary couplings frequently occur between residues whose side chains point towards one another. Compared to the side-chain center based method, $\mathrm{C} \alpha$ - (and to a lesser extent $\mathrm{C} \beta$-) based contact definitions classify a smaller number of contacts in this orientation and this is likely the cause of decreased predictive accuracies that we observe when using these latter methods to define contacts (Fig. 4).

\section{DISCUSSION}

The co-evolutionary patterns of amino acid substitutions can provide important information about protein structures. A number of competing methods are currently in use to detect evolutionary couplings between residues, and the ability to recover true residue-residue contacts has been the primary metric employed to assess performance of various methods. However, true structural contacts are ill-defined and variability in contact definitions can prohibit comparison between the efficacy of different methods as well as obscure
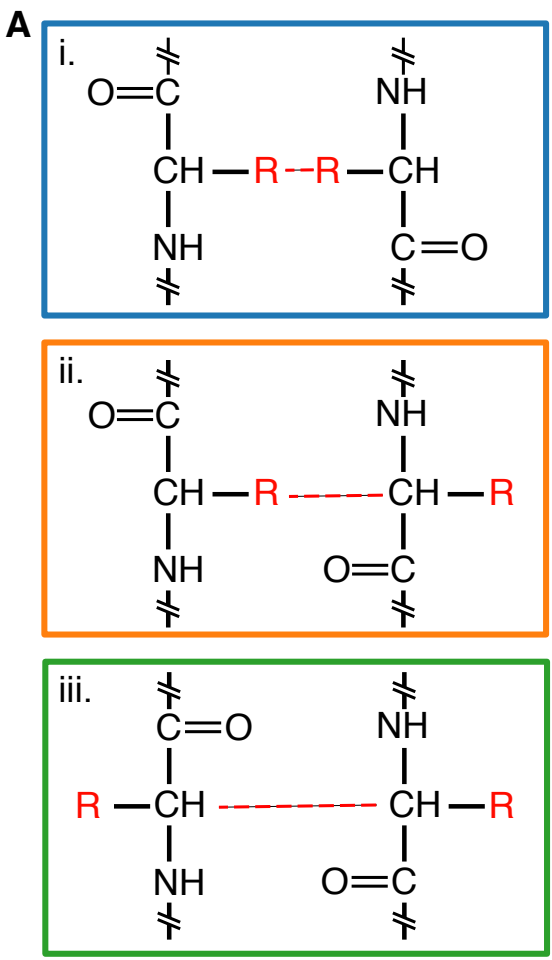

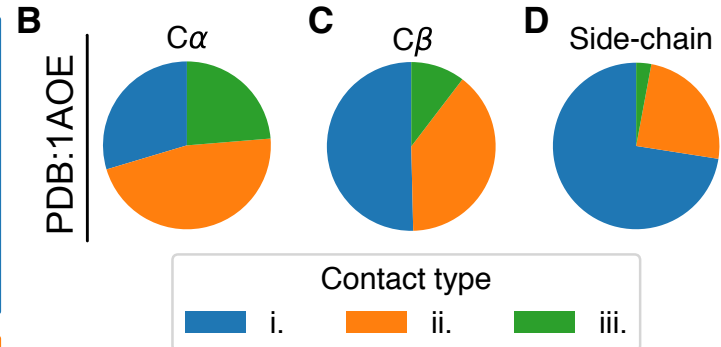

E

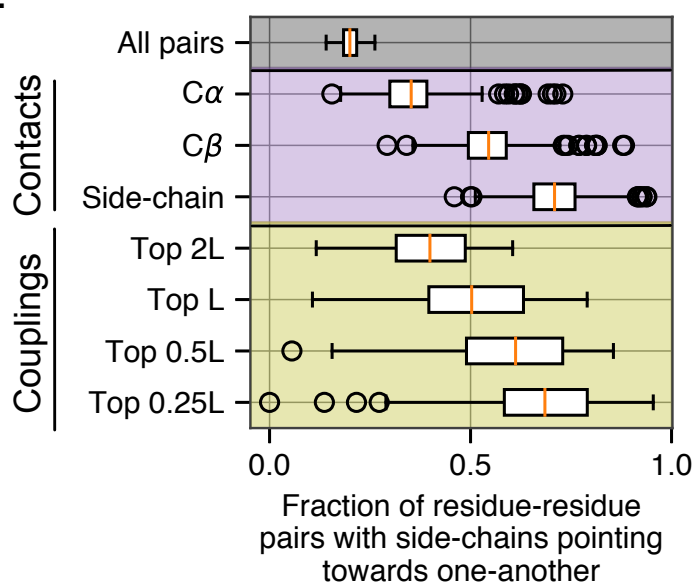

Figure 5. Different types of residue-residue interactions are possible. (A) Two interacting residues may interact via: each residue's side-chain atoms (type i), the side chain of one residue and the backbone of the other (type ii), or the backbone atoms of each residue (type iii). (B-D) For intramolecular contacts identified in PDB:1AOE, the relative proportion of different interaction types varies according to contact identification method. Shown are contacts identified via $\mathrm{C} \alpha$ atoms $(B), \mathrm{C} \beta$ atoms $(C)$, and side-chain centers $(D)$; residue-residue contacts defined via side-chain centers are enriched in type i interactions (blue). (E) For 150 proteins, the fraction of residue-residue pairs where the side chains point towards one-another is highest in contacts defined via side-chain centers (purple). Further, the top ranked evolutionary couplings are progressively enriched in residue-residue pairs where the side chains point towards one another (yellow). 
the biological interpretation of evolutionary constraints. We have shown here that evolutionary couplings are significantly more accurate at detecting true residue-residue contacts defined via side-chain center distances compared to $\mathrm{C} \alpha$ or $\mathrm{C} \beta$ distances. This finding provides important biological insight into protein evolution and epistatic interactions between residues. We posit that although different types of interactions between amino acid residues may stabilize protein structures, evolutionary couplings predominantly represent residues whose contact occurs via interactions between the side-chain atoms of both residues.

While we have shown that using side-chain centers as a reference point to define intramolecular contacts is advantageous compared to $\mathrm{C} \alpha$ or $\mathrm{C} \beta$ atoms, it is important to note that the contacts defined via side-chain centers are still likely to be only a rough approximation of reality. Actual contacts between residues within a single structure would ideally be defined via knowledge of the hydrogen bonding, van der Waals forces, and ionic/dipole/hydrophobic interactions that collectively stabilize protein structures (Abdel-Azeim et al., 2014; Doshi et al., 2016; Mercadante et al., 2018). Defining contacts via these features can be time-consuming and resource intensive and the use of distance-based cutoffs is therefore advantageous under many circumstances. Furthermore, a highly accurate view of residue-residue contacts may still not be particularly useful or informative. For instance, our findings do not suggest that contacts between the backbone atoms of residues do not occur or that they are unimportant for stabilizing protein structures. Rather, we show that contacts between backbone atoms are not likely to be detected by evolutionary coupling analyses. Thus, regardless of the method used for defining residue-residue contacts, different contact types may be of varying importance for different applications.

Amongst distance-based methods, a potential disadvantage of using side-chain centers as reference points is that the geometric center of different amino acid side chains lies at varying absolute distances from the peptide backbone. Here, we have used arbitrary distance cutoffs to define side-chain center contacts (based on identifying an equal number of contacts per protein as an $8 \AA \mathrm{C} \alpha$ cutoff). This approach facilitates comparison of different contact identification methods on the same scale, but a more rigorous approach would be preferable. In practice, we have found that a distance cutoff of $7.5 \AA$ for side-chain centers works well, and we thus recommend this approach moving forward (SI Fig. S8), although the physical justification for such a cutoff is unclear. The method of evaluating the minimum distance between side-chain heavy atoms is particularly attractive in this regard, since it permits using a physically realistic and uniform distance cutoff between atoms regardless of amino acid identity or the protein under consideration. While it is possible that analysis of still larger datasets might uncover differences in accuracies between these conceptually similar approaches, our study shows that these methods produce nearly identical results and the choice between them should be based on the tradeoff between simplicity of calculation (side-chain centers) and physical reality (minimum atomic distances between side-chain atoms with a uniform cutoff).

Leveraging homologous sequence information to predict intramolecular protein contacts has long been a goal of structural biologists, but progress towards this goal has been made possible only in recent years with increasing availability of sequence information and the development and application of Direct Coupling Analysis to isolate directly interacting residue-residue correlations from spurious correlation generated by chains of directly coupled residues (Burger and Van Nimwegen, 2008, 2010; Jones et al., 2012; Ekeberg et al., 2013; Seemayer et al., 2014). However, there are still a number of known limitations to current methods for computing evolutionary couplings as illustrated by the variability in performance that we observe when applying the same basic algorithms to collect and evaluate different homologous protein sets (Fig. 4A). Most evolutionary coupling algorithms require large numbers of sequence homologs to produce accurate predictions (Ovchinnikov et al., 2017); we observed this in our own data, where the number of sequences in a multiple sequence alignment is significantly correlated with the PPV of inferred evolutionary couplings (SI Fig. S4). However, the size of the multiple sequence alignment explains only a moderate fraction of the variation in accuracies. Features such as the evolutionary relatedness of sequences and the heterogeneity of substitution rates across sites may impose further constraints on the overall identifiability of evolutionary couplings, but it is not clear whether these effects should systematically vary between different protein families (Vorberg et al., 2018).

False positive predictions resulting from evolutionary coupling analyses may arise from a combination of different factors including problems with repeat proteins, homo-oligomerization, and structural variation within protein families (Anishchenko et al., 2017). Here, we have shown that another source of false positives in evolutionary coupling analyses may simply be ill-defined true positive contacts. We also observed that accuracy improvements stemming from the use of side-chain centers as a reference point 
were variable across proteins (Fig. 4C,E) - this variability may be related to features of the proteins themselves in terms of their compactness or other physio-chemical properties.

Practically speaking, contact identification methods and evolutionary couplings are increasingly important for a variety of applications. Frequently, evolutionary couplings are combined with a variety of other features and used as input for machine learning and associated neural network-based algorithms to predict structural and functional properties of proteins (Cheng and Baldi, 2007; Tegge et al., 2009; Lena et al., 2012; Jones et al., 2015; Michel et al., 2017; Xiong et al., 2017; Stahl et al., 2017; He et al., 2017; Wang et al., 2017; Riesselman et al., 2018; Liu et al., 2018; Wozniak et al., 2018; Jones and Kandathil, 2018; Adhikari et al., 2018; Hanson et al., 2018). Our analysis suggests that there may be biases in the training data - essential to supervised learning techniques - owing to the method used to define true positive contacts. Given that raw evolutionary couplings more accurately predict contacts identified by side-chain centers, we speculate that the accuracy of supervised learning algorithms may be similarly improved by training and testing on contacts identified via side-chain distances. Different contact types can be classified according to which atoms interact between residue-residue pairs (Fig. 5A), and it is possible that the accuracy of supervised approaches in particular may be improved by separating different types of residue-residue contacts according to their atomic interactions, training separate models to detect each type, and integrating the results.

\section{CONCLUSIONS}

Sequence based co-evolutionary methods are a powerful tool for studying structural and functional constraints on protein families, and the accuracy of these methods is frequently benchmarked by their ability to predict residue-residue contacts. Here, we show that there are numerous ways to summarize a given protein structure as a set of residue-residue contacts and that the choice of how to do this mapping has important consequences for downstream applications. We find that the predictions of evolutionary coupling algorithms are substantially more accurate when predicting residue-residue contacts defined via their side chains, highlighting the important role that side-chain interactions play in governing epistasis and protein evolution. Based on these results, we recommend defining residue-residue contacts via either side-chain centers (as a fixed reference point) or the minimum atomic distances between side-chain atoms for applications moving forward.

\section{Acknowledgements}

The authors acknowledge valuable feedback and support from members of the Wilke lab.

\section{REFERENCES}

Abdel-Azeim, S., Chermak, E., Vangone, A., Oliva, R., and Cavallo, L. (2014). MDcons : Intermolecular contact maps as a tool to analyze the interface of protein complexes from molecular dynamics trajectories. BMC Bioinformatics, 15:1-11.

Adhikari, B., Hou, J., and Cheng, J. (2018). DNCON2: Improved protein contact prediction using two-level deep convolutional neural networks. Bioinformatics, 34(9):1466-1472.

Amunts, A., Brown, A., Bai, X.-c., Llacer, J. L., Hussain, T., Emsley, P., Long, F., Murshudov, G., Scheres, S. H., and Ramakrishnan, V. (2014). Structure of the Yeast Mitochondrial Large Ribosomal Subunit. Science, 343:1485-1489.

Anfinsen, C. B. (1973). Principles that Govern the Folding of Protein Chains. Science, 181(4096):223-230.

Anishchenko, I., Ovchinnikov, S., Kamisetty, H., and Baker, D. (2017). Origins of coevolution between residues distant in protein 3D structures. Proceedings of the National Academy of Sciences, page 201702664.

Batyuk, A., Galli, L., Ishchenko, A., Han, G. W., Gati, C., Popov, P. A., Lee, M.-Y., Stauch, B., White, T. A., Barty, A., Aquila, A., Hunter, M. S., Liang, M., Boutet, S., Pu, M., Liu, Z.-j., Nelson, G., James, D., Li, C., Zhao, Y., Spence, J. C. H., Liu, W., Fromme, P., Katritch, V., Weierstall, U., Stevens, R. C., and Cherezov, V. (2016). Native phasing of X-ray free-electron laser data for a G protein-coupled receptor. Science Advances, 2(9).

Bhattacharya, D. and Cheng, J. (2013). 3Drefine: Consistent protein structure refinement by optimizing hydrogen bonding network and atomic-level energy minimization. Proteins: Structure, Function and Bioinformatics, 81(1):119-131. 
Biasini, M., Bienert, S., Waterhouse, A., Arnold, K., Studer, G., Schmidt, T., Kiefer, F., Cassarino, T. G., Bertoni, M., Bordoli, L., and Schwede, T. (2014). SWISS-MODEL: Modelling protein tertiary and quaternary structure using evolutionary information. Nucleic Acids Research, 42:252-258.

Burger, L. and Van Nimwegen, E. (2008). Accurate prediction of protein-protein interactions from sequence alignments using a Bayesian method. Molecular Systems Biology, 4(165).

Burger, L. and Van Nimwegen, E. (2010). Disentangling direct from indirect co-evolution of residues in protein alignments. PLoS Computational Biology, 6(1).

Chaudhury, S., Lyskov, S., and Gray, J. J. (2010). PyRosetta: A script-based interface for implementing molecular modeling algorithms using Rosetta. Bioinformatics, 26(5):689-691.

Chen, C., Natale, D. A., Finn, R. D., Huang, H., Zhang, J., Wu, C. H., and Mazumder, R. (2011). Representative Proteomes: A Stable, Scalable and Unbiased proteome set for sequence analysis and functional annotation. PLOS ONE, 6(4):e18910.

Cheng, J. and Baldi, P. (2007). Improved residue contact prediction using support vector machines and a large feature set. BMC Bioinformatics, 8(1).

Denisov, I. G. and Sligar, S. G. (2016). Nanodiscs for structural and functional studies of membrane proteins. Nature Structural and Molecular Biology, 23(6):481-486.

Doncheva, N. T., Klein, K., Domingues, F. S., and Albrecht, M. (2011). Analyzing and visualizing residue networks of protein structures. Trends in Biochemical Sciences, 36(4):179-182.

Doshi, U., Holliday, M. J., Eisenmesser, E. Z., and Hamelberg, D. (2016). Dynamical network of residue-residue contacts reveals coupled allosteric effects in recognition, catalysis, and mutation. Proc Natl Acad Sci USA, 113(17):4735-4740.

Duarte, J. M., Sathyapriya, R., Stehr, H., Filippis, I., and Lappe, M. (2010). Optimal contact definition for reconstruction of Contact Maps. BMC Bioinformatics, 11.

Ekeberg, M., Lövkvist, C., Lan, Y., Weigt, M., and Aurell, E. (2013). Improved contact prediction in proteins: Using pseudolikelihoods to infer Potts models. Physical Review E, 87(1):1-16.

Figliuzzi, M., Barrat-Charlaix, P., and Weigt, M. (2018). How pairwise coevolutionary models capture the collective residue variability in proteins? Molecular Biology and Evolution, 35(4):1018-1027.

Gobel, U., Sander, C., Schneider, R., and Valencia, A. (1994). Correlated Mutations and Residue Contacts in Proteins. Proteins, 18:309-317.

Hanson, J., Paliwal, K., Litfin, T., Yang, Y., and Zhou, Y. (2018). Accurate Prediction of Protein Contact Maps by Coupling Residual Two-Dimensional Bidirectional Long Short-Term Memory with Convolutional Neural Networks. Bioinformatics, 34(23):4039-4045.

He, B., Mortuza, S. M., Wang, Y., Shen, H. B., and Zhang, Y. (2017). NeBcon: Protein contact map prediction using neural network training coupled with naïve Bayes classifiers. Bioinformatics, 33(15):2296-2306.

Hopf, T. A., Colwell, L. J., Sheridan, R., Rost, B., Sander, C., and Marks, D. S. (2012). Three-dimensional structures of membrane proteins from genomic sequencing. Cell, 149(7):1607-1621.

Hopf, T. A., Green, A. G., Schubert, B., Mersmann, S., Schärfe, C. P., Ingraham, J. B., Toth-Petroczy, A., Brock, K., Riesselman, A. J., Palmedo, P., Kang, C., Sheridan, R., Draizen, E. J., Dallago, C., Sander, C., and Marks, D. S. (2018). The EVcouplings Python framework for coevolutionary sequence analysis Thomas. Bioinformatics, page bty862.

Hopf, T. A., Ingraham, J. B., Poelwijk, F. J., Schärfe, C. P., Springer, M., Sander, C., and Marks, D. S. (2017). Mutation effects predicted from sequence co-variation. Nature Biotechnology, 35(2):128-135.

Hopf, T. A., Scharfe, C. P. I., Rodrigues, J. P. G. L. M., Green, A. G., Kohlbacher, O., Sander, C., Bonvin, A. M. J. J., and Marks, D. S. (2014). Sequence co-evolution gives 3D contacts and structures of protein complexes. eLife, 3:1-45.

Jiang, Q., Teufel, A. I., Jackson, E. L., and Wilke, C. O. (2018). Beyond Thermodynamic Constraints: Evolutionary Sampling Generates Realistic Protein Sequence Variation. Genetics, 208:1387-1395.

Jones, D. T., Buchan, D. W., Cozzetto, D., and Pontil, M. (2012). PSICOV: Precise structural contact prediction using sparse inverse covariance estimation on large multiple sequence alignments. Bioinformatics, 28(2):184-190.

Jones, D. T. and Kandathil, S. M. (2018). High precision in protein contact prediction using fully convolutional neural networks and minimal sequence features. Bioinformatics, 34(19):3308-3315.

Jones, D. T., Singh, T., Kosciolek, T., and Tetchner, S. (2015). MetaPSICOV: Combining coevolution methods for accurate prediction of contacts and long range hydrogen bonding in proteins. Bioinformatics, 
31(7):999-1006.

Kamisetty, H., Ovchinnikov, S., and Baker, D. (2013). Assessing the utility of coevolution-based residueresidue contact predictions in a sequence- and structure-rich era. Proceedings of the National Academy of Sciences, 110(39):15674-15679.

Katoh, K. and Standley, D. M. (2013). MAFFT multiple sequence alignment software version 7: Improvements in performance and usability. Molecular Biology and Evolution, 30(4):772-780.

Kellogg, E. H., Leaver-Fay, A., and Baker, D. (2011). Role of conformational sampling in computing mutation-induced changes in protein structure and stability. Proteins, 79(3):830-838.

Lapedes, A. S., Giraud, B. G., Liu, L., and Stormo, G. D. (1999). Correlated mutations in models of protein sequences: phylogenetic and structural effects. Statistics in molecular biology and genetics, 33:236-256.

Leaver-Fay, A., Tyka, M., Lewis, S. M., Lange, O. F., Thompson, J., Jacak, R., Kaufman, K., Renfrew, P. D., Smith, C. A., Sheffler, W., Davis, I. W., Cooper, S., Treuille, A., Mandell, D. J., Richter, F., Ban, Y. E. A., Fleishman, S. J., Corn, J. E., Kim, D. E., Lyskov, S., Berrondo, M., Mentzer, S., Popović, Z., Havranek, J. J., Karanicolas, J., Das, R., Meiler, J., Kortemme, T., Gray, J. J., Kuhlman, B., Baker, D., and Bradley, P. (2011). Rosetta3: An object-oriented software suite for the simulation and design of macromolecules. Methods in Enzymology, 487(Chapter 19):545-574.

Lena, P. D., Nagata, K., and Baldi, P. (2012). Deep architectures for protein contact map prediction. Bioinformatics, 28(19):2449-2457.

Liao, M., Cao, E., Julius, D., and Cheng, Y. (2013). Structure of the TRPV1 ion channel determined by electron cryo-microscopy. Nature, 504(7478):107-112.

Lin, C. P., Huang, S. W., Lai, Y. L., Yen, S. C., Shih, C. H., Lu, C. H., Huang, C. C., and Hwang, J. K. (2008). Deriving protein dynamical properties from weighted protein contact number. Proteins, 72(3):929-935.

Liu, G., Shen, Y., Atreya, H. S., Parish, D., Shao, Y., Sukumaran, D. K., Xiao, R., Yee, A., Lemak, A., Bhattacharya, A., Acton, T. A., Arrowsmith, C. H., Montelione, G. T., and Szyperski, T. (2005). NMR data collection and analysis protocol for high-throughput protein structure determination. Proceedings of the National Academy of Sciences, 102(30):10487-10492.

Liu, Y., Palmedo, P., Ye, Q., Berger, B., and Peng, J. (2018). Enhancing Evolutionary Couplings with Deep Convolutional Neural Networks. Cell Systems, 6(1):65-74.e3.

Marcos, M. L. and Echave, J. (2015). Too packed to change: side-chain packing and site-specific substitution rates in protein evolution. PeerJ, 3:e911.

Marks, D. S., Colwell, L. J., Sheridan, R., Hopf, T. A., Pagnani, A., Zecchina, R., and Sander, C. (2011). Protein 3D structure computed from evolutionary sequence variation. PLoS ONE, 6(12).

Marks, D. S., Hopf, T. A., and Sander, C. (2012). Protein structure prediction from sequence variation. Nature Biotechnology, 30(11):1072-1080.

Mercadante, D., Gräter, F., and Daday, C. (2018). CONAN: A tool to decode dynamical information from molecular interaction maps. Biophysical Journal, 114:1267-1273.

Miao, J., Ishikawa, T., Robinson, I. K., and Murnane, M. M. (2015). Beyond crystallography: Diffractive imaging using coherent x-ray light sources. Science, 348(6234):530-535.

Michel, M., Skwark, M. J., Hurtado, D. M., Ekeberg, M., and Elofsson, A. (2017). Predicting accurate contacts in thousands of Pfam domain families using PconsC3. Bioinformatics, 33(18):2859-2866.

Morcos, F., Pagnani, A., Lunt, B., Bertolino, A., Marks, D. S., Sander, C., Zecchina, R., Onuchic, J. N., Hwa, T., and Weigt, M. (2011). Direct-coupling analysis of residue coevolution captures native contacts across many protein families. Proceedings of the National Academy of Sciences, 108(49):E1293-E1301.

Ovchinnikov, S., Kamisetty, H., and Baker, D. (2014). Robust and accurate prediction of residue-residue interactions across protein interfaces using evolutionary information. eLife, pages 1-21.

Ovchinnikov, S., Park, H., Varghese, N., Huang, P.-S., Pavlopoulos, G. A., Kim, D. E., Kamisetty, H., Kyrpides, N. C., and Baker, D. (2017). Protein structure determination using metagenome sequence data. Science, 355(6322):294-298.

Piovesan, D., Minervini, G., and Tosatto, S. C. (2016). The RING 2.0 web server for high quality residue interaction networks. Nucleic acids research, 44(W1):W367-W374.

Potter, S. C., Luciani, A., Eddy, S. R., Park, Y., Lopez, R., and Finn, R. D. (2018). HMMER web server: 2018 update. Nucleic Acids Research, 46(W1):W200-W204.

Punjani, A., Rubinstein, J. L., Fleet, D. J., and Brubaker, M. A. (2017). CryoSPARC: Algorithms for 
rapid unsupervised cryo-EM structure determination. Nature Methods, 14(3):290-296.

Riesselman, A. J., Ingraham, J. B., and Marks, D. S. (2018). Deep generative models of genetic variation capture mutation effects. Nature Methods.

Rose, P. W., Prlić, A., Altunkaya, A., Bi, C., Bradley, A. R., Christie, C. H., Di Costanzo, L., Duarte, J. M., Dutta, S., Feng, Z., Green, R. K., Goodsell, D. S., Hudson, B., Kalro, T., Lowe, R., Peisach, E., Randle, C., Rose, A. S., Shao, C., Tao, Y. P., Valasatava, Y., Voigt, M., Westbrook, J. D., Woo, J., Yang, H., Young, J. Y., Zardecki, C., Berman, H. M., and Burley, S. K. (2017). The RCSB protein data bank: Integrative view of protein, gene and 3D structural information. Nucleic Acids Research, 45:D271-D281.

Sadowski, M. I. and Jones, D. T. (2009). The sequence-structure relationship and protein function prediction. Current Opinion in Structural Biology, 19(3):357-362.

Sathyapriya, R., Duarte, J. M., Stehr, H., Filippis, I., and Lappe, M. (2009). Defining an essence of structure determining residue contacts in proteins. PLoS Computational Biology, 5(12).

Scarabelli, G. and Grant, B. J. (2013). Mapping the structural and dynamical features of kinesin motor domains. PLoS Computational Biology, 9(11).

Schaarschmidt, J., Monastyrskyy, B., Kryshtafovych, A., and Bonvin, A. M. (2018). Assessment of contact predictions in CASP12: Co-evolution and deep learning coming of age. Proteins: Structure, Function and Bioinformatics, 86(October 2017):51-66.

Seeliger, D. and de Groot, B. L. (2007). Atomic contacts in protein structures. A detailed analysis of atomic radii, packing, and overlaps. Proteins, 68:595-601.

Seemayer, S., Gruber, M., and Söding, J. (2014). CCMpred - Fast and precise prediction of protein residue-residue contacts from correlated mutations. Bioinformatics, 30(21):3128-3130.

Serçinoğlu, O. and Ozbek, P. (2018). gRINN: A tool for calculation of residue interaction energies and protein energy network analysis of molecular dynamics simulations. Nucleic Acids Research, 46(W1):W554-W562.

Shahmoradi, A. and Wilke, C. O. (2016). Dissecting the roles of local packing density and longer-range effects in protein sequence evolution. Proteins, 84(6):841-854.

Shindyalov, I. N., Kolchanov, N. A., and Sander, C. (1994). Can three-dimensional contacts in protein structures be predicted by analysis of correlated mutations? Protein Engineering, Design and Selection, 7(3):349-358.

Söding, J. (2005). Protein homology detection by HMM-HMM comparison. Bioinformatics, 21(7):951960.

Stahl, K., Schneider, M., and Brock, O. (2017). EPSILON-CP: Using deep learning to combine information from multiple sources for protein contact prediction. BMC Bioinformatics, 18(1):1-11.

Sulkowska, J. I., Morcos, F., Weigt, M., Hwa, T., and Onuchic, J. N. (2012). Genomics-aided structure prediction. Proceedings of the National Academy of Sciences, 109(26):10340-10345.

Tegge, A. N., Wang, Z., Eickholt, J., and Cheng, J. (2009). NNcon: Improved protein contact map prediction using 2D-recursive neural networks. Nucleic Acids Research, 37:515-518.

Teufel, A. I. and Wilke, C. O. (2017). Accelerated simulation of evolutionary trajectories in origin-fixation models. Journal of the Royal Society Interface, 14(127).

Vorberg, S., Seemayer, S., and Söding, J. (2018). Synthetic protein alignments by CCMgen quantify noise in residue-residue contact prediction. PLoS Computational Biology, 14(11):e1006526.

Wang, S., Sun, S., Li, Z., Zhang, R., and Xu, J. (2017). Accurate De Novo Prediction of Protein Contact Map by Ultra-Deep Learning Model. PLoS Computational Biology, 13(1):e1005324.

Wang, S., Sun, S., and Xu, J. (2018). Analysis of deep learning methods for blind protein contact prediction in CASP12. Proteins: Structure, Function and Bioinformatics, 86(August 2017):67-77.

Weigt, M., White, R. A., Szurmant, H., Hoch, J. A., and Hwa, T. (2009). Identification of direct residue contacts in protein-protein interaction by message passing. Proceedings of the National Academy of Sciences, 106(1):67-72.

Wozniak, P. P., Pelc, J., Skrzypecki, M., Vriend, G., and Kotulska, M. (2018). Bio-knowledge based filters improve residue- residue contact prediction accuracy. Bioinformatics, 34(21):3675-3683.

Wyman, S. K., Avila-herrera, A., Nayfach, S., and Id, S. P. (2018). A most wanted list of conserved microbial protein families with no known domains. PLoS One, 13(10):e0205749.

Xiong, D., Zeng, J., and Gong, H. (2017). A deep learning framework for improving long-range residue-residue contact prediction using a hierarchical strategy. Bioinformatics, 33(17):2675-2683. 
624 Yuan, C., Chen, H., and Kihara, D. (2012). Effective inter-residue contact definitions for accurate protein 625 fold recognition. BMC Bioinformatics, 13(1). 